\section{EDUCAÇÃO EM SAÚDE SOBRE VULVOVAGINITES PARA MULHERES ATENDIDAS EM UM CENTRO DE SAÚDE DA FAMÍLIA}

\author{
Health education on vulvovaginitis for women seen at a family \\ health center
}

\author{
Educación para la salud sobre vulvovaginitis en mujeres \\ asistidas en un centro de salud de la familia
}

\begin{abstract}
RESUMO
Objetivo: Desenvolver ações educativas, visando à construção de conhecimento acerca das vulvovaginites, identificando o conhecimento adquirido e o desejo de mudança de atitude após as ações. Métodos: Pesquisa descritiva e qualitativa, realizada no Centro de Saúde da Família (CSF) Maria Adeodato, no município de Sobral-CE, no período de março a maio de 2013. Realizaram-se 10 ações educativas sobre vulvovaginites com mulheres atendidas no CSF, sendo abordadas temáticas diferentes. Cada ação foi estruturada em três momentos: diagnóstico do conhecimento prévio, ação educativa com o conteúdo a ser trabalhado e avaliação após a intervenção. A coleta de informações ocorreu por meio de entrevista semiestruturada, para identificação das participantes, e grupo focal, para as etapas de diagnóstico e avaliação, sendo os conteúdos analisados por meio de categorias temáticas. Resultados: Foram contempladas oito participantes, com idade entre 48 e 55 anos, escolaridade entre $1^{\circ}$ ano do ensino fundamental e fundamental completo, a maioria solteira, com no mínimo cinco filhos, e que não realizavam o exame ginecológico com frequência. As categorias de análise geradas foram denominadas de "conhecimento das mulheres sobre o corpo e a sua higiene íntima", "prevenção e controle de vulvovaginites" e "percepção das mulheres sobre exame ginecológico". Percebeu-se que as participantes do estudo desconheciam a terminologia "vulvovaginite", bem como sinais, sintomas e meios de transmissão da doença, ou tinham apenas um breve conhecimento sobre o assunto. Conclusão: Após as ações educativas, identificou-se a aquisição de conhecimento pelas participantes e expressões que indicaram o desejo de mudança.
\end{abstract}

Descritores: Vulvovaginite; Exame Ginecológico; Educação em Saúde.

\section{ABSTRACT}

Objective: To develop educational activities, aiming at the construction of knowledge about vulvovaginitis, identifying the knowledge acquired and the desire for attitude change after the activities. Methods: A qualitative descriptive research, held at Maria Adeodato Family Health Center (FHC) in the city of Sobral, Ceará, in the period from March to May 2013. Ten educational activities regarding vulvovaginites were performed with women seen at the FHC, addressing different topics. Every action was divided into three phases: diagnosis of prior knowledge, educational activities with the subject to be covered, and afterwards evaluation. Data was collected through semi-structured interview, for the participant's identification, and focal group, for the diagnostic and evaluation phases, and content analysis was performed using thematic categories. Results: The research comprised 8 participants, aged between 48 and 55 years, with schooling ranging from the first year of primary education to complete primary education, mostly single, having a minimum of 5 children, and the participants did not undergo the gynecological exam frequently. The generated analysis categories were described as 'women's knowledge of the body (genitals) and their intimate hygiene', 'prevention and control of vulvovaginites' and 'women's perception about gynecological exam'. It was evidenced that the study participants ignored the terminology 'vulvovaginitis' as well as the disease signs, symptoms, and means of transmission, or just had a brief knowledge of the subject. Conclusion: After the educational activities, the study identified knowledge acquirement by the participants and expressions indicating the desire for changing.

Descriptors: Vulvovaginitis; Gynecological Examination; Health Education.
Artigo Original

$$
\begin{array}{r}
\text { Renata Soares Morais } \\
\text { Maria Eliane de Sousa } \\
\text { Albuquerque }
\end{array}
$$

Samy Loraynn Oliveira Moura ${ }^{(1)}$ Germana Maria da Silveira ${ }^{(1)}$ Marcelo dos Santos Feitoza ${ }^{(1)}$ Denise Tomaz Aguiar ${ }^{(1)}$

1) Universidade Estadual Vale do Acaraú UVA - Sobral (CE) - Brasil

Recebido em: 26/11/2013

Revisado em: 25/03/2014 Aceito em: 01/10/2014 


\section{RESUMEN}

Objetivo: Desarrollar acciones educativas para la construcción del conocimiento sobre las vulvovaginitis, identificando el conocimiento adquirido y el deseo de cambio de actitud después de las acciones. Métodos: Investigación descriptiva y cualitativa realizada en el Centro de Salud de la Familia (CSF) Maria Adeodato en el municipio de Sobral-CE entre marzo y mayo de 2013. Se realizaron 10 acciones educativas sobre vulvovaginitis en mujeres asistidas en el CSF donde se han trabajado distintas temáticas. Cada acción fue estructurada en tres momentos: diagnóstico del conocimiento previo, acción educativa con el contenido a ser trabajado y evaluación después de la intervención. La recogida de las informaciones se dio a través de entrevista semi-estructurada para identificación de las participantes y grupo focal para las fases de diagnóstico y evaluación con sus contenidos analizados en categorías temáticas. Resultados: Ocho participantes con edad entre 48 y 55 años, escolaridad en el primer año de la educación primaria y educación primaria completa, la mayoría soltera con un mínimo de cinco hijos y que no realizaban el examen ginecológico con frecuencia. Las categorías de análisis fueron: "conocimiento de las mujeres sobre el cuerpo y su higiene intima", "prevención y control de vulvovaginitis" y "percepción de las mujeres sobre el examen ginecológico". Se percibió que las participantes del estudio no conocian la terminología "vulvovaginitis", asi como los síntomas y las formas de transmisión de la enfermedad o sabian poco del asunto. Conclusión: Después de las acciones educativas, se identificó la adquisición del conocimiento de las participantes y expresiones que sugieren el deseo de cambio.

Descriptores: Vulvovaginitis; Examen Ginecológico; Educación en Salud.

\section{INTRODUÇÃO}

Dentre as patologias que acometem a mulher, destacam-se aquelas que denominamos genericamente de vulvovaginites, caracterizadas por um processo infeccioso e/ou inflamatório vulvovaginal, causadas principalmente por bactérias, fungos ou vírus ${ }^{(1)}$.

De acordo com o Ministério da Saúde, vulvovaginite é toda manifestação inflamatória e/ou infecciosa do trato genital feminino inferior, ou seja, vulva, vagina e epitélio escamoso do colo uterino (ectocérvice). O quadro clínico é variável de acordo com a etiologia, podendo manifestarse pela presença de corrimento vaginal, dor, irritação ou prurido. Entretanto, muitas infecções genitais podem ser completamente assintomáticas ${ }^{(2)}$. A vulvovaginite é uma das causas mais comuns de corrimento vaginal patológico e responsável por inúmeras consultas aos ginecologistas ${ }^{(3)}$.

A enfermagem tem um papel fundamental no controle das vulvovaginites, seja desenvolvendo atividades de promoção da saúde; intervindo individualmente, na família ou na comunidade; detectando fatores e situações de risco; promovendo educação em saúde; contribuindo para o diagnóstico precoce, a adesão e o tratamento efetivo do paciente e seu parceiro sexual. Além disso, responsabilizase pelo acolhimento do paciente, cuidando ou coordenando outros setores para a prestação de assistência ${ }^{(4)}$.

O interesse pelo tema surgiu durante o acompanhamento das consultas de enfermagem, nas quais se percebeu a prevalência das vulvovaginites nos resultados dos exames citopatológicos das mulheres que procuraram o serviço naquele período.

No intuito de contribuir para amenizar a problemática citada, este estudo objetivou desenvolver ações educativas, visando à construção de conhecimento acerca das vulvovaginites, identificando o conhecimento adquirido e o desejo de mudança de atitude após as ações.

\section{MÉTODOS}

Trata-se de uma pesquisa descritiva, com abordagem qualitativa, que permite estabelecer uma estrutura coletiva, participativa e ativa, possibilitando uma maior interação das pessoas envolvidas.

O período do estudo compreendeu os meses de março a maio de 2013, tendo como cenário o Centro de Saúde da Família (CSF) Maria Adeodato, localizado no bairro da Expectativa, em Sobral-CE. O município de Sobral fica no sertão centro-oeste do Estado do Ceará a $238 \mathrm{~km}$ da capital Fortaleza. Atualmente, de acordo com o senso 2011 do Instituto Brasileiro de Geografia e Estatística (IBGE), Sobral possui 188.271 habitantes ${ }^{(5)}$.

O CSF é composto por duas equipes de Estratégia de Saúde da Família (ESF), com 5 enfermeiros, 21 agentes comunitários de saúde, 4 auxiliares de enfermagem, 2 médicos, 1 fisioterapeuta, 1 educador físico, 1 assistente social, 1 psicólogo, 1 dentista e 1 atendente de dentista, que acolhem as necessidades de 3.400 famílias residentes na área de abrangência.

Participaram do estudo oito mulheres, com idade entre 40 e 60 anos, participantes de um grupo do referido CSF que se reúne para discutir assuntos referentes à saúde da mulher. O grupo existe há um ano e seis meses, sendo coordenado por quatro agentes comunitárias de saúde, sob a supervisão de uma enfermeira da unidade. É composto por 10 participantes, entretanto, duas delas estavam ausentes em função de problemas pessoais.

Para as participantes, foram considerados como critérios de seleção: residir na área do referido CSF, comparecer às reuniões do grupo de mulheres e aceitar participar do estudo, assinando o Termo de Consentimento Livre e Esclarecido (TCLE). 
Inicialmente, visitou-se o CSF, a fim de conhecer melhor o cenário de estudo e as participantes do grupo de mulheres.

Realizaram-se 10 encontros, sendo o primeiro com o objetivo de acolher as mulheres do grupo e proporcionar uma maior aproximação com os autores. Nesse momento, pactuou-se o dia, horário e periodicidade do grupo, aplicando a entrevista e solicitando a assinatura do TCLE. Além disso, utilizando entrevistas estruturadas, coletaramse informações referentes à caracterização socioeconômica e ao conhecimento prévio das mulheres sobre vulvovaginites. Para avaliar o conhecimento prévio, foram feitas as seguintes perguntas: o que deve ser feito na prevenção e no cuidado com doença na vagina? Qual a importância que você atribui ao exame, acompanhamento e orientação quanto aos cuidados e prevenção de doenças na vulva e na vagina? Sobre quais assuntos você gostaria de conversar?

No segundo encontro, conversou-se sobre a anatomia feminina, trazendo também um pouco sobre sexualidade, por meio de material audiovisual. No terceiro encontro, abordou-se a temática da higiene íntima. O quarto encontro teve como temática o exame de prevenção do câncer ginecológico, fazendo uma demonstração de como o exame ocorre, com uma prótese feminina e o material utilizado.

No quinto encontro, discorreu-se sobre câncer de mama através de próteses mamárias e material audiovisual. No sexto encontro, falou-se sobre vulvovaginites por meio de uma roda de conversa sobre o assunto, com o auxílio de material audiovisual. No sétimo encontro, conversouse sobre algumas doenças sexualmente transmissíveis, sendo entregue um folder para as participantes. No oitavo encontro, a pedido das participantes, discutiu-se sobre hábitos saudáveis para a melhoria da qualidade de vida. No nono encontro, abordou-se a importância do autocuidado físico e mental, fazendo-se uso de material audiovisual e técnicas de relaxamento. Para finalizar, o décimo encontro foi para a avaliação de todos os momentos vivenciados até então.

Para a análise dos dados, utilizou-se o método de categorias temáticas, as quais, operacionalmente, dividemse em três etapas: pré-análise, exploração do material, e tratamento dos resultados obtidos e interpretação ${ }^{(6)}$. Dessa forma, a partir da leitura e releitura do material, chegouse à construção de categorias temáticas, nominadas de "conhecimento das mulheres sobre o corpo (genitália) e a sua higiene íntima", "prevenção e controle de vulvovaginites" e "percepção das mulheres sobre exame ginecológico".

Vale ressaltar que foram respeitados os princípios éticos presentes na Resolução 466/12(7) do Conselho Nacional de Saúde. Dessa forma, foi garantido o anonimato das mulheres, sendo identificadas pela letra P (participante), seguida de um número distribuído aleatoriamente (P1, P2...). O estudo obteve parecer favorável da Comissão Científica da Secretaria de Saúde e Ação Social de Sobral-CE, e do Comitê de Ética em Pesquisa da Universidade Estadual Vale do Acaraú (UVA), com parecer n 352.804/13.

\section{RESULTADOS E DISCUSSÃO}

As participantes do estudo tinham idade entre 48 e 55 anos, a escolaridade variou do $1^{\circ}$ ano do ensino fundamental ao fundamental completo, sendo em sua maioria solteiras, com no mínimo cinco filhos, e não realizavam o exame ginecológico com frequência.

A partir das entrevistas realizadas, das sessões educativas e da análise do material produzido, apresentamse, a seguir, os dados referentes às categorias de análise.

Conhecimento das mulheres sobre o corpo (genitália) e a sua higiene íntima

$\mathrm{Na}$ oficina sobre higiene íntima, percebeu-se, ao mostrar a imagem da vulva e vagina, o desconhecimento e espanto de algumas mulheres a respeito da própria genitália.

Vixe, é é assim? (P6)

Nem lembrava mais como era. (P4)

E a vagina é a parte de dentro, por fora é a vulva? Valha! (P5)

$\mathrm{O}$ atendimento integral à saúde feminina é de grande importância, e os profissionais de saúde devem estimular a mulher a assumir o próprio cuidado, pois a imagem que ela tem sobre o funcionamento interno do corpo influencia a percepção dos eventos e a experiência corporal ${ }^{(8,9)}$.

Observou-se, em um estudo, que a mulher não é estimulada por profissionais de saúde, ou mesmo pela mídia, a fazer o toque vaginal ou conhecer a genitáliai ${ }^{(9)}$, fato notado e expresso pelas depoentes desta pesquisa quando indagadas se já olharam ou olham com frequência sua genitália no espelho:

\section{Essas coisas ninguém vê não, é feio. (P4)}

Quem quer ver isso no espelho? (P6)

Vixe! Não e não! (P3)

Apesar do desconhecimento das mulheres em relação ao seu corpo, identificou-se que, após a ação, houve um aumento do conhecimento e uma tomada de novas iniciativas a respeito do assunto, observado pelas falas a seguir:

Assim que chegar em casa, eu vou entrar no quarto, pegar um espelho e ver as coisas direitinho, para se acostumar com ela. (P4) 
Agora eu sei. Chegar em casa, [vou] olhar a bichinha direitinho. (P5)

A educação em saúde significa contribuir para que as pessoas adquiram autonomia, com a finalidade de identificar e utilizar as formas e os meios para preservar e melhorar a sua vida. Assim, uma boa ação educativa tem por objetivo levar conhecimento e provocar uma mudança de atitude ${ }^{(10)}$.

No intuito de compreender como as participantes realizavam a higiene íntima, indagou-se sobre o que "pode" ou " não pode" ser feito para se ter uma higiene íntima adequada. Houve concordância em alguns pontos e dúvidas em outros.

\section{Vixe! Dormir sem calcinha pode não, tá errado. (P1)}

Pois eu durmo é sem calcinha, às vezes até fico em casa sem calcinha e acho é bom. (P6)

Perfume? Num pode colocar lá que arde, tem que deixar $o$ 'cheirim' natural dela. (P2)

Pode usar sabonete íntimo sempre. (P7)

Roupa apertada 'num' pode, que esquenta e depois dá doença. (P8)

De acordo com a Federação Brasileira das Associações de Ginecologia e Obstetrícia (FEBRASGO), a oclusão pelo uso de roupas íntimas, absorventes higiênicos e produtos de higiene inadequados torna a pele da vulva suscetível a várias dermatoses ${ }^{(11)}$.

Além disso, orienta-se que a higienização anogenital feminina apenas com água não permite a remoção de resíduos e secreções acumulados nessa região, sendo necessário o uso de produtos de limpeza que tenham alguma detergência associados em sua fórmula, para facilitar a emulsificação das gorduras e a remoção das partículas microscópicas de papel, células mortas da pele, urina, fezes e sangue menstrual ${ }^{(11)}$.

\section{Prevenção e controle de vulvovaginites}

Inicialmente, procurou-se captar o conhecimento que as participantes apresentavam sobre o assunto. Para tanto, desenvolveu-se uma roda de conversa, a fim de que pudessem relatar o que entendiam por vulvovaginite.

Nunca ouvi falar não. 'Num'sei o que é. (P3)

Não, tenho nem ideia. (P6)

É aquele negócio que dá coceira, que suja a calcinha, da calcinha mal lavada, que tem que passar o ferro na calcinha para não ter. (P7)

Vixe! Sei nada não. (P1)

Com relação às vulvovaginites, percebeu-se que as participantes do estudo desconhecem a terminologia, bem como sinais, sintomas e meios de transmissão das doenças, ou apenas têm um breve conhecimento sobre o assunto em um estudo no Centro de Saúde Escola do município de Botucatu-SP(12).

Logo em seguida, fez-se uma explanação sobre o tema, sendo identificado que a maioria das participantes já havia apresentado algum sintoma de vulvovaginite.

Eu já tive muito corrimento quando eu era nova, 'num'sei se era da quentura, porque eu trabalhava muito no sol, ai eu tinha muito corrimento, coceira, ai eu fui me consultar e o médico passava remédio. (P5)

Eu tinha direto dor no pé da barriga e muita coceira. (P8)

A partir dos relatos, indagou-se sobre os cuidados que poderiam ser estabelecidos para tratar e/ou prevenir a ocorrência de tais patologias.

\section{Não andar descalço na terra quente. (P5)}

Deve procurar o médico [...], fazer exames, sexo com camisinha. (P2)

Só se eliminar totalmente as relações sexuais. (P1)

Lavar com casca de aroeira na água morna, com ameixa e casca de consales. (P3)

Folha de malva passada no liquidificador, ai faz o banho de assento junto com a casca de aroeira. (P8)

Em outro estudo realizado, também se observou que as mulheres utilizavam plantas medicinais no cuidado íntimo, principalmente pela questão financeira, pois são mais baratas do que os medicamentos alopáticos ${ }^{(13)}$.

\section{Percepção das mulheres sobre exame ginecológico}

Por meio de dinâmicas e conversa aberta, discorreu-se sobre a importância do exame ginecológico para a prevenção do câncer de colo do útero e, com o auxílio de prótese e materiais utilizados no exame, explicou-se como ele é feito, em uma linguagem clara e simples. Nesse momento, identificou-se que as participantes não realizavam o exame ginecológico com frequência.

Faz tempo que eu não faço meu exame de prevenção! (P6) Vou confessar que às vezes a gente precisa de alguém para lembrar de cuidar da nossa saúde. (P7)

O exame é desconfortável, mas a gente tem que se cuidar, é importante. (P1)

Dá medo de fazer, mas tem que fazer. (P2)

Como encontrado em outro estudo, as mulheres veem o exame ginecológico como algo incômodo, porém necessário e importante; como uma forma de cuidar de si mesma, ou seja, apesar da insegurança e do medo do exame, elas o têm como algo necessário para a prevenção e o diagnóstico de problemas $^{(14)}$.

Percebe-se, então, como são importantes as ações de educação em saúde, para que se possa esclarecer 
dúvidas e proporcionar uma melhor eficácia nos métodos de prevenção de patologias. Durante o encontro, as participantes mostraram-se interessadas em aprender, fizeram muitos questionamentos e relataram o desejo em mudar seus hábitos.

Assim que terminar a reunião, vou marcar meu exame. (P5)

A gente tem que se cuidar, porque senão tem uma doença mais séria. (P7)

Eu aprendi muita coisa hoje. [...] Vou me cuidar mais. (P3)

Observou-se que as questões relacionadas à genitália feminina ainda são pouco abordadas com as mulheres usuárias do sistema de saúde. Porém, as ações de educação em saúde se mostraram como uma estratégia importante para esclarecer dúvidas, proporcionar uma melhor eficácia nos métodos de prevenção de patologias e instigar o desejo de mudança de hábitos.

\section{CONSIDERAÇÕES FINAIS}

A partir do estudo, observou-se o desconhecimento das mulheres em relação às questões ligadas ao seu corpo, em especial ao seu cuidado íntimo. Após as ações educativas, identificou-se a aquisição de conhecimento pelas participantes e expressões que indicaram o desejo de mudança.

É necessário, portanto, que haja continuidade na discussão dessas questões com as usuárias do Sistema Único de Saúde, com vistas ao desenvolvimento de uma atitude de autocuidado e à quebra de paradigmas envolvendo o tema. Ressalta-se, ainda, a necessidade da ampliação de pesquisas desenvolvendo essa temática e envolvendo a educação em saúde, a fim de contribuir para formar uma nova visão sobre o trabalho preventivo e de cuidado à saúde da mulher.

\section{REFERÊNCIAS}

1. Cavalcante VLN, Miranda AT, Portugal GMP. Rastreamento de Candidose Vaginal Durante a Prevenção do Câncer Cérvico-Uterino. DST J Bras Doenças Sex Transm. 2005;17(1):44-8.

2. Ministério da Saúde (BR), Secretaria de Atenção à Saúde. Controle dos cânceres do colo do útero e da mama. Brasília: Ministério da Saúde; 2006. (Cadernos de Atenção Básica, n. 13)

3. Federação Brasileira das Associações de Ginecologia e Obstetrícia - FEBRASGO, Jacyntho C. Manual de orientação em trato genital inferior e colposcopia: vulvovaginites. São Paulo: FEBRASGO; 2010.
4. Rocha SMM, Almeida MCP. O processo de trabalho da enfermagem em saúde coletiva e a interdisciplinaridade. Rev Latinoam Enferm. 2000;8(6):96-101.

5. Instituto Brasileiro de Geografia e Estatística IBGE.Cidades@: informações sobre os municípios brasileiros. Brasília: IBGE; 2011 [acesso em 2014 Jun 1]. Disponível em: http://estados.ibge.gov.br/estadosat/ perfil.php?sigla $=$ ce

6. Minayo MCS. O desafio do conhecimento: pesquisa qualitativa em saúde. $11^{\mathrm{a}}$ ed. São Paulo: Hucitec; 2008.

7. Ministério da Saúde (BR). Resolução CNS no 466/12. Dispõe sobre diretrizes e normas regulamentadoras de pesquisas envolvendo seres humanos. Brasília: Conselho Nacional de Saúde; 2012.

8. Costa GMC, Gualda DMR. Conhecimento e significado cultural da menopausa para um grupo de mulheres. Rev Esc Enferm USP. 2008;42(1):81-9.

9. Salimena AMO, Coelho ACP, Melo MCSC, Greco RM, Almeida MIG. Conhecimentos e atitudes de mulheres varredoras de rua sobre o cuidado ginecológico. Texto \& Contexto Enferm. 2012;21(1):43-51.

10. Oliveira HM, Gonçalves MJF. Educação em saúde: uma experiência transformadora. Rev Bras Enferm. 2004;57(6):761-3.

11. Federação Brasileira das Associações de Ginecologia e Obstetrícia - FEBRASGO. Guia de Condutas sobre higiene genital feminina. São Paulo: FEBRASGO; 2009.

12. Soares MT. Percepção de mulheres sobre consulta de enfermagem, exame de Papanicolau e vulvovaginites [trabalho de conclusão de curso]. São Paulo: Faculdade de Medicina de Botucatu; 2010 [acesso em 2014 Jan 28]. Disponível em: http://base.repositorio.unesp.br/ handle/11449/121349

13. Leitão EF, Costa LLS, Brêda MZB, Albuquerque MCS, Jorge JS. A prática cotidiana de saúde das profissionais do sexo. Rev Bras Promoç Saúde. 2012;25(3):295-304.

14. Andrade SSC, Silva FMC, Silva MSS, Oliveira SHS, Leite KNS, Sousa MJ. Compreensão de usuárias de uma Unidade de Saúde da Família sobre o exame Papanicolaou. Ciênc Saúde Coletiva. 2013;18(8): 2301-10.

\section{Endereço para correspondência:}

Renata Soares Morais

Universidade Estadual Vale do Acaraú - UVA

Centro de Ciências da Saúde

Avenida da Universidade, 850 - Campus da Betânia

CEP: 62.040-370 - Sobral - CE - Brasil 